\title{
RESPONSABILIDAD SOCIAL EEMPRESARIAL (RSE) Y SU COMUNICACIÓN EN LAS PYME
}

\author{
CSR and its communication in SMEs \\ RSE e sua comunicação em Pmes
}

\author{
Pilar Flores-Asenjo \\ Universidad Catolica San Antonio de Murcia \\ pflores@ucam.edu \\ Miguel Angel Beltran-Bueno \\ Universidad Catolica San Antonio de Murcia \\ mabeltran@ucam.edu
}

\section{Resumo}

Através da análise e síntese da recente literatura sobre RSC (responsabilidade social corporativa) e RSE (responsabilidade social empresarial), principalmente em PMEs, pretendese realizar uma taxonomia das diferentes formas de assumir a RSC e as características, motores e limitações das mesmas, para que sirva como um instrumento para as instituições na elaboração de estratégias de divulgação e manutenção da RSE.

Palavras-chave: RSC. Comunicação estratégica. Estratégia empresarial.

\begin{abstract}
Through the review and synthesis of the recent literature on CSR and CSR, especially in SMEs, it is intended to make taxonomy of the different ways of assuming SR and the characteristics, motivations and limitations of the implementation of social responsibility, to serve as an instrument to the institutions in the design of strategies for the increase and maintenance of CSR.
\end{abstract}

Key words: CSR. Strategy communication. Business strategy.

\section{Resumen}

Mediante la revisión y síntesis de la literatura reciente sobre RSC (responsabilidad social corporativa) y RSE (responsabilidad social empresarial), principalmente en las pymes, se pretende realizar una taxonomía de las distintas formas de asumir la RS y las características, motores y limitaciones de las mismas, para que sirva como instrumento a las instituciones en el diseño de estrategias de difusión y mantenimiento de la RSE.

Palabras clave: RSC. Comunicación estratégica. Estrategia empresarial. 


\section{INTRODUCCIÓN: DEFINICIÓN DE RSC Y RSE}

Un desafío frecuentemente señalado es la falta de definición o perspectiva comúnmente aceptada de la RSC (responsabilidad social corporativa). Los estudios revelan amplios significados y prácticas bajo el nombre de SRC o RSE (responsabilidad social empresarial).

La RSE se entiende a menudo como un compromiso ético por parte de la empresa para responder a un "Triple Bottom Line" social y medioambiental, así como a los objetivos económicos de sostenibilidad (ELKINGTON, 1997; SAVITZ y WEBER, 2006). Las prácticas asociadas con la RSE pueden incluir la transparencia en la rendición de cuentas, el respeto de los valores éticos, la mejora de la calidad de vida, la preservación de los entornos naturales, el apoyo a los esfuerzos comunitarios locales, así como abordar cuestiones de información sobre privacidad, derechos de propiedad intelectual, libertad de expresión, y la calidad y fiabilidad de la información facilitada por la empresa (DHILLON, 2002).

Las distintas definiciones dadas en la literatura tienen como puntos en común la "voluntariedad de las acciones" (MARTÍNEZ y AGÜERO, 2008), la integración de preocupaciones sociales y ambientales en la política de la empresa y la orientación hacia las interacciones con todos los “grupos de interés". Así, la definición de RSC propuesta por la Comisión de las Comunidades Europeas (CE) en 2011 (una de las más aceptadas) indica que "estas empresas integran las preocupaciones sociales y medioambientales en sus operaciones empresariales y en sus interacciones con sus partes interesadas de forma voluntaria".

En el caso concreto de la RSC en las PYME, diversos estudios sugieren que el lenguaje actual y los debates para la empresa socialmente responsable (SR), así como su comprensión y prescripción pueden requerir una reconceptualización para las pequeñas empresas (MADUEÑO et al., 2014; CRANE, et al.,2013; LANGE y FENWICK, 2007; JAMALI et al., 2008; JENKINS, 2004). El término RSE parece más adecuado para las PYMES (SCHAPER y SAVERY, 2004; CÁSTKA et al., 2004; JENKINS, 2006; BESSERA y MILLER, 2001; SWEENY, 2009), pues el término "corporativo" puede ser asimilado a la gran empresa.

Así, Castka et al. (2004, p.148) propusieron una definición para las PYMES en la que la RSE se define como "un medio para dirigir las organizaciones de manera rentable, pero de 
forma social y ambientalmente responsable, para lograr la sostenibilidad del negocio y la satisfacción de las partes interesadas".

\section{MARCO TEÓRICO}

La naturaleza multidisciplinar de la RSE ha llevado al desarrollo de varias teorías en un intento de entender sus diversas dimensiones. Frynas y Yamahaki (2016) y Mason y Simmons (2014), por ejemplo, señalaron que la corriente principal de la investigación en RSE está dominada en gran medida por las teorías relacionales, especialmente la teoría de "los grupos de interés”. Sin embargo, otras investigaciones han hecho hincapié en el lado aplicado de la gestión ya que consideran que las ideas de RSE sólo pueden desarrollarse mediante procesos de planificación y dirección estratégica (JONKER y de WITTE, 2006; LINDGREEN et al., 2009).

En todo caso, aparecen dos formas distintas de adaptar la RSE: una más estratégica y relacionada con los las relaciones de mercado y el beneficio de la empresa (PORTER y KRAMER, 2006; VIRVILAITE y DAUBARAITE, 2015), y otra más altruista, relacionada con las orientaciones humanitarias de cuidado, reciprocidad e incluso responsabilidad global que pueden o no recoger beneficios materiales para la empresa (KELL, 2003).

Spence y Rutherfoord (2000) propusieron que quienes adoptan un enfoque pasivo de la RSE tienen más probabilidades de priorizar la supervivencia o la maximización de los beneficios, mientras que las PYMES que buscan activamente la RSE están más inclinadas a ser socialmente responsables, creyendo que "haciendo las cosas bien" obtendrán mejores resultados a largo plazo.

Los que siguen la orientación estratégica indican que la clave de la adopción es la capacidad organizativa estratégica: en lugar de adoptar prácticas excepcionales, el éxito de la RS depende de la implementación de "un patrón sistemático de prácticas simples pero múltiples, coherentes y coordinadas que simultáneamente reduzcan los impactos ambientales y los costos de organización" (ARAGÓN CORREA et al. 2008: 99). Para Jenkins (2006), la implementación exitosa depende de la integración de responsabilidad social (RS) en todos los aspectos de la práctica empresarial, no como un complemento externo, sino como núcleo de la operación del negocio.

En este caso, la responsabilidad social corporativa (RSC) o responsabilidad social empresarial (RSE), como compromiso integral de una empresa u organización de contribuir al 
desarrollo y bienestar de la calidad de vida de la comunidad, puede generar oportunidades, innovación y ventaja competitiva para la empresa, y además contribuir al bienestar social de la comunidad. La justificación instrumental de la RSE que da como resultado una ventaja competitiva es ahora ampliamente aceptada por los académicos y los empresarios (CARROLL y SHABANA, 2010).

Sin embargo, a pesar del reciente cambio de los argumentos basados en la ética a un enfoque instrumental, algunos autores continúan argumentando que el enfoque ético sigue siendo una justificación fundamental para la RSE (SPENCE y SCHMIDPETER, 2003; LEE et al., 2015), en términos de promover el bien común (McELHANEY, 2008).

Son muchos los autores que han analizado la importancia de las PYMES en la economía y, por tanto, la importancia de la RSE en este contexto. La justificación se debe a que más del $99 \%$ del total de organizaciones son de tamaño pequeño y a que aportan las dos terceras partes del empleo europeo (GARCÍA-BORBOLLA et al., 2009).

Em cambio, la adopción de prácticas socialmente responsables no ha tenido un desarrollo similar entre las grandes corporaciones y las pequeñas y medianas empresas debido fundamentalmente a las diferencias propias de éstas últimas en cuanto a la estructura de la propiedad, acceso a los recursos, características del empresario, sus relaciones con los grupos de interés y el ámbito local donde operan, entre otras (JENKINS, 2004; CILIBERTI et al, 2008).

Según algunos autores, sus especiales características pueden facilitar el proceso de RSE dado que, por su tamaño poseen una mayor flexibilidad para adaptarse con rapidez a los cambios de un mercado complejo, globalizado y cada vez más competitivo y pueden responder con mayor facilidad a las demandas de sus grupos de interés (stakeholders) o construir ventajas competitivas en nichos de mercado que incorporen beneficios ambientales y/o sociales (JENKINS, 2006; SWEENEY, 2007; RIVES y BAÑÓN, 2008).

Un cierto compromiso de las PYMES con la RSE es el resultado de la presión del mercado o de las partes interesadas (SEIDEL et al., 2008; GALLARDO-VÁZQUEZ et al., 2013). Otro motivo es la creencia de que si las empresas adoptan prácticas de RSE, obtendrán beneficios inmediatos o a largo plazo de sostenibilidad y rentabilidad para sus empresas (CARROLL y SHABANA, 2010), tales como mejores relaciones con las partes interesadas internas y externas, motivación del personal y lealtad del cliente (LEE et al., 2012; JENKINS, 2006), ventaja competitiva a través de la diferenciación, posibilidades de innovación (PORTER y KRAMER, 2006; TANTALO et al., 2012), y ganar capital social mediante el 
apoyo a la comunidad local (RUSSO y PERRINI, 2010). Las PYMES pueden reducir residuos y ahorrar costes y conseguir una mejor reputación como empresa responsable (KECHICHE y SOPARNOT, 2012).

Otros autores, en cambio, indican que los beneficios percibidos de la RSC entre los propietarios y gerentes de las PYMES están mucho más alineados con las dimensiones altruistas como la participación, el sentimiento de orgullo y la integridad que con los beneficios propiamente económicos (JENKINS 2006). Los factores que contribuyen a esta motivación pueden incluir la integración de las PYMES dentro de sus comunidades locales y sus estrechas relaciones personales con proveedores, comunidades, socios y empleados que configuran la ética y el comportamiento de los propietarios o gerentes de las PYMES (VIVES, 2006; MURILLO y LOZANO, 2006; PERRINI, 2006).

El poder se distribuye más uniformemente en las organizaciones más grandes entre los gerentes de varios departamentos, mientras que, en las PYMES, el propietario generalmente tiene una gran influencia en la mayoría de las decisiones estratégicas (SPENCE, 1999). Por lo tanto, los valores y creencias personales del propietario-gerente de la PYME tienen una profunda influencia en el compromiso de RSC dentro de la empresa (MURILLO y LOZANO, 2006; JAMALI et al., 2009; JENKINS, 2004 y 2006; LEPOUTRE y HEENE, 2006; SPENCE y RUTHERFOORD, 2000). Además, la menor separación entre propiedad y control favorecería la visualización de los incentivos que puede ofrecer para la empresa la consecución de prácticas que satisfagan intereses de los diversos stakeholders (WEBB, 2004). Las PYMES se orientan principalmente hacia la solución de problemas cotidianos, donde las relaciones y la comunicación son en su mayoría informales, y las relaciones interpersonales se consideran importantes. Las PYMES están altamente interrelacionadas con su entorno o comunidades en las que operan y, a menudo, actúan como proveedores de empresas más grandes, en cuyo caso están sujetas a la dinámica de mercado determinada por ellas (MURILLO y LOZANO, 2006). Algunas evidencias sugieren que las PYMES dependen de las relaciones con los stakeholders porque la legitimidad de su negocio es directamente responsable ante la comunidad local: una de las principales motivaciones para el comportamiento de RS puede ser la lealtad del cliente y la reputación del negocio local (FULLER y TIAN, 2006; KUSYK y LOZANO, 2007).

Cuando se aplica a la RSE, la evidencia empírica indica que algunas PYMES diseñan y administran sus prácticas de RSC en torno a sus diferentes grupos de interesados, tales como programas relacionados con empleados y clientes (JENKINS, 2006; LEE et al., 2012). 
Los investigadores han identificado tres obstáculos principales para la implementación exitosa de las prácticas de RSE por parte de las PYMES: el escepticismo de los propietariosadministradores sobre la RSC, la falta de conocimiento y las limitaciones de recursos.

Puesto que la supervivencia empresarial sigue siendo considerada como la principal preocupación de muchas PYMES, algunos gestores de las mismas consideran que la responsabilidad social de las empresas no es responsabilidad suya, mientras que otros sostienen que su contribución a los impactos sociales y medioambientales no sería significativa.

Otra razón de la baja aceptación de la RSE por parte de las PYMES es la falta de conocimiento sobre lo que implica el concepto de RSE y cómo deben implementarse las prácticas de RSC. Varios estudios han identificado que la falta de conocimiento sobre cómo implementar eficazmente las prácticas de RSE en la empresa es otro obstáculo para las PYMES (MAON et al., 2009).

Las PYMES a menudo se enfrentan a más dificultades que las grandes empresas cuando tratan de involucrarse en la RSE, debido principalmente a limitaciones de recursos. Las principales barreras para las PYMES preocupadas por las cuestiones sociales y ambientales son las restricciones en las finanzas, los recursos humanos y el tiempo (JENKINS, 2004; LEPOUTRE y HEENE, 2006).

Otro factor diferenciador es la comunicación. La comunicación de la RSC es una parte importante de la gestión de la RSE, ya que es mediante una comunicación eficaz con las diferentes partes interesadas que una empresa puede lograr mejor sus objetivos de RSC y obtener beneficios estratégicos de sus actividades de RSE; sin embargo, algunos estudios revelan que la conciencia de los esfuerzos de RSE de una empresa entre sus partes interesadas internas y externas suele ser baja (DU et al., 2010). Las PYMES llevan a cabo una responsabilidad social silenciosa (ARGANDOÑA, 2008; JAMALI et al. 2008; RUSSO, y TENCATI, 2008), gestionan un gran número de impactos económicos, sociales y medioambientales, pero no utilizan el lenguaje de la RSE para describirlas (JENKINS, 2004; LONGO et al., 2005; PERRINI, 2006).

A diferencia de las grandes empresas, las PYMES, al operar en mercados locales y depender en mayor medida de fuentes internas para financiar su crecimiento, tienen menor presión en la formalización de sus prácticas. En general, se caracterizan por tener una menor documentación de sus operaciones y por aplicar un estilo de gestión informal a las cuestiones estratégicas y la comunicación (RUSSO y TENCATI, 2009; PANG et al., 2011). 
Las investigaciones demuestran que existe una necesidad urgente de que las empresas encuentren maneras de comunicar la RSE de manera más eficaz y adecuada a fin de mejorar las relaciones de los interesados, lo que puede dar lugar a mejores beneficios empresariales (LEE et al., 2015; DU et al., 2010).

\section{METOdOLOGÍA: PROPUESTA DE CLASIFICACIÓN DE EMPRESAS CON RSE}

Según las características de las empresas analizadas en la literatura sobre RSE y RSC, se puede establecer una taxonomía de las mismas atendiendo a la motivación para su implantación, el nivel de RSE desarrollado y su integración en la planificación estratégica y en la comunicación de la empresa:

A partir de la motivación para implantar RSE, podemos clasificar a las empresas en aquellas cuya iniciativa tuvo su fundamento o raíz en posiciones altruistas o éticas (necesidad de hacer las cosas bien y/o de aportar algo bueno al entorno o la sociedad) y las que implantan acciones de RSE como medio de obtener una ventaja competitiva o un beneficio económico (mejorar la reputación o imagen de la empresa para generar mayores ingresos o lealtad de los clientes, aumentar la productividad del personal mejorando el clima laboral, reducir costes energéticos...). Además, estas actividades pueden focalizarse en acciones sociales o medioambientales.

Por otra parte, las empresas pueden desarrollar o no su RSE como parte de su planificación estratégica y, por tanto, llevar a cabo una comunicación formal o informal de las mismas. En este sentido, muchas de las PYMES analizadas por los distintos trabajos sobre RSE llevan a cabo una "RSE silenciosa", que se correspondería con unas acciones no estructuradas/planificadas y una comunicación informal de las mismas a sus "grupos de interés". 


\begin{tabular}{|c|c|c|c|c|}
\hline & & & \multicolumn{2}{|c|}{$\begin{array}{l}\text { Planificación estratégica y } \\
\text { comunicación }\end{array}$} \\
\hline & & & $\begin{array}{l}\text { Estructurada y } \\
\text { Formal }\end{array}$ & $\begin{array}{l}\text { No estructurada } \\
\text { e Informal }\end{array}$ \\
\hline \multirow{4}{*}{$\begin{array}{l}\text { Motivación } \\
\text { para implantar } \\
\text { RSE }\end{array}$} & \multirow{2}{*}{ Altruista } & Social & $\begin{array}{l}\text { Concienciada } \\
\text { social }(\mathrm{E} 1)\end{array}$ & $\begin{array}{l}\text { Proactiva social } \\
\text { (E5) }\end{array}$ \\
\hline & & Medioambiental & $\begin{array}{l}\text { Concienciada } \\
\text { ambiental (E2) }\end{array}$ & $\begin{array}{l}\text { Proactiva } \\
\text { ambiental (E6) }\end{array}$ \\
\hline & \multirow{2}{*}{ Competitiva } & Social & $\begin{array}{l}\text { Reactiva social } \\
(\mathrm{E} 3)\end{array}$ & $\begin{array}{l}\text { Adaptativa } \\
\text { social (E7) }\end{array}$ \\
\hline & & Medioambiental & $\begin{array}{l}\text { Reactiva } \\
\text { ambiental (E4) }\end{array}$ & $\begin{array}{l}\text { Adaptativa } \\
\text { ambiental (E8) }\end{array}$ \\
\hline
\end{tabular}

Tabla 1 - Tipología de empresas com RSE

Fuente: Elaboración propia

\section{DISCUSIÓN Y RESULTADOS DE LA PROPUESTA DE CLASIFICACIÓN DE EMPRESAS CON RSE}

Las empresas tipo E1 incluyen la RSE en su planificación estratégica y su comunicación es formal, además, establecieron la RSE de forma consciente con motivos éticos o altruistas, y centrados en las acciones sociales: mejoras de la calidad de vida, integración de la mujer en el mundo laboral, conciliación familiar, ayuda a la comunidad local, etc. Son empresas que suelen poseer algún tipo de certificación, maduras, con suficientes recursos (financieros y humanos) y una relación de comunicación participativa con los grupos de interés.

Las empresas tipo E2, en cambio, orientan la mayoría de sus actividades de RSE a la mejora del medioambiente. Generalmente, suelen pertenecer a un sector de actividad muy relacionado con el medio, y sus certificaciones se orientan al desarrollo sostenible de su actividad dentro de él. Estas empresas implantan la RSE, no como respuesta al mercado, sino por convencimiento de que es "lo que se debe hacer", aunque esto a largo plazo les lleva a desarrollar ventajas competitivas y focalizar los esfuerzos de innovación en este tipo de nicho de mercado.

Las empresas tipo E1 y E2 podrían denominarse empresas "concienciadas". Son empresas en las que la RSE está contenida en la propia dirección estratégica de la empresa (misión y visión) y desarrollan sus actividades realizando una comunicación formal, multidireccional y participativa de las mismas con los grupos de interés.

Las empresas tipo E3 y E4, en cambio, implantan la RSE como reacción a sus relaciones con los "grupos de interés", buscando un beneficio económico a corto plazo. Son 
empresas que utilizan certificaciones sociales o ambientales y comunicaciones para mejorar la imagen y reputación de la empresa de cara a sus clientes o proveedores, principalmente, o en sus relaciones con las instituciones y administraciones.

Estas empresas "reactivas", realizan sus actividades de RSE de forma estructurada y su comunicación es formal y bidireccional (con el grupo de interés que ha llevado a la empresa a involucrarse en las actividades).

Las empresas tipo E5 y E6, "proactivas", se diferencian de las E1 y E2 en que desarrollan una RSE “silenciosa”. Llevan a cabo acciones sociales y/o ambientales porque la dirección imprime sus valores éticos en el funcionamiento diario de la empresa. Los propietarios/directivos reconocen los conflictos éticos relacionados con lo que es una acción "correcta" como propietario de una pequeña empresa y cuál es el grado justificable de su responsabilidad con su ideal de RS elegido. Sus estrategias se desarrollan a través de un proceso de aprendizaje que está arraigado en las negociaciones en curso dentro de la acción cotidiana y sus interactuaciones. La responsabilidad social puede estudiarse más fructíferamente en estas empresas examinando las huellas de estas redes, los vínculos y los límites formulados a través de la interacción cotidiana.

Las empresas tipo E7 y E8, "adaptativas”, implantan acciones ante las presiones de los grupos de interés o las oportunidades del mercado, siempre y cuando les genere un beneficio por el aumento de ingresos o reducción de los costes (medidas medioambientales de eficiencia energética, reducción de multas por incumplimiento de normativas...). Sin embargo, debido a insuficiencia de recursos (financieros, tiempo y formación), desarrolla las actividades de forma no estructurada y su comunicación es informal y unidireccional (al grupo de interés con mayor repercusión en su actividad).

Mayoritariamente, las PYMES se encontrarán reflejadas, según la literatura analizada, en los tipos E5, E6, E7 y E8 debido a que, por regla general, cuentan con recursos financieros limitados y sus sistemas o procedimientos internos se desarrollan de manera informal (JENKINS, 2004). Algunos autores indican que predominan las E7 y E8, ya que las PYMES no consideran favorablemente la RSE si es poco probable que las inversiones sean compensadas en términos de ahorro de costes (KECHICHE y SOPARNOT, 2012) o incremento de ingresos. Otros, en cambio, opinan que en las PYMES el propietario generalmente tiene una gran influencia en la mayoría de las decisiones estratégicas (SPENCE, 1999), por lo tanto, los valores y creencias personales del propietario-gerente de la PYME tienen una profunda influencia en el compromiso de RSC dentro de la empresa (MURILLO y 
LOZANO, 2006; JAMALI et al., 2009; JENKINS, 2004 y 2006; LEPOUTRE y HEENE, 2006; SPENCE y RUTHERFOORD, 2000). De esta manera, los beneficios percibidos de la RSC entre los propietarios y gerentes de las PYMES estarían mucho más alineados con las dimensiones altruistas como la participación, el sentimiento de orgullo y la integridad que con los beneficios propiamente económicos (Jenkins 2006), predominando, por tanto, los tipos E6 y E5. Esto puede deberse en parte a que, como sostiene Spence (2007), no hay pruebas sólidas de que la práctica de la RSE conduzca al éxito financiero y a la ventaja competitiva, o simplemente no sea percibido por los propietarios.

\section{CONCLUSIONES}

Si entendemos la Responsabilidad Social Corporativa (RSC) entre las PYME, como la “integración voluntaria por parte de las empresas, de las preocupaciones sociales y medioambientales en sus operaciones comerciales y en sus relaciones con sus interlocutores" (COMISIÓN de las COMUNIDADES EUROPEAS -CCE-, 2001, pp. 7), la RSC engloba un conjunto de acciones de formalización de sistemas de gestión, de comunicación y de transparencia informativa que han de ser analizados para determinar la forma y grado de implantación de las mismas y, por tanto, sus motores y las barreras para diseñar una buena estrategia de promoción.

Las especiales características de las PYME frente a las grandes empresas han creado un debate acerca de la motivación principal de implantación de RSE entre los que defienden el rol de la misión y visión estratégica de la empresa (representada por los valores éticos de los propietarios/gerentes) y los que explican la implantación por las oportunidades de logro de ventajas competitivas en el mercado. Esta dualidad ha sido analizada en las empresas a través de la teoría de "relaciones de los grupos de interés" y las "estrategias competitivas".

El análisis empírico llevado a cabo por distintos autores en diferentes regiones del mundo ha apoyado una y otra teoría, dando lugar a una tipología diferente de empresas en función de la naturaleza de la motivación de implantación de RSE y del tipo de comunicación e implantación estratégica dentro de la misma.

La síntesis de los estudios analizados ha dado lugar a una taxonomía de empresas en las que queda claro el origen de la RSE, el tipo de implantación estratégica y su comunicación, con lo que es fácil identificar motores y barreras que pueden afectar a su desarrollo. Así, encontramos las empresas “concienciadas”, que tienen la RSE completamente 
integrada en sus estrategias y comunicación, y que se fundaron bajo una visión y misión en la que los valores éticos motivaron dicha implicación. Las empresas "reactivas" en cambio, a pesar de haber conseguido integrar la RSE en sus estrategias y comunicación, tienen como principal motor o motivador el "logro de ventajas competitivas" y un "buen clima de relación con sus grupos de interés". La mayoría de las PYME, sin embargo, no tiene integradas las acciones de RSE dentro de su planificación estratégica y llevan una RSE "silenciosa", así, nos encontramos con empresas "proactivas", que realizan acciones de RSE porque el propietario/gerente cree que es "como debe de actuar" y las "adaptativas" que realizan las acciones para lograr un beneficio (reducción de costes, incremento de la productividad laboral...).

La tarea del sector público hacia la RSC se define a menudo como la de "crear un entorno facilitador" y servir de guía para que las empresas y la sociedad adopten prácticas más responsables socialmente. Para diseñar estrategias eficientes para ayudar a la implantación y mantenimiento de RSE, teniendo en cuenta sus especiales características, motores y limitaciones, resulta interesante conocer el tipo de empresas que predominan en la economía, pues los lazos entre las iniciativas empresariales y las políticas regionales, en el marco de una comunicación constante con organizaciones sociales, se revelan como indispensables para asegurar el éxito de la RSE.

\section{REFERÊNCIAS}

ARAGÓN-CORREA, J. A., HURTADO-TORRES, N., SHARMA, S., y GARCÍA-MORALES, V. J. Environmental strategy and performance in small firms: A resource-based perspective. Journal of environmental management, 86(1), pp. 88-103, 2008.

ARGANDOÑA, A. La responsabilidad social de las empresas pequeñas y medianas. Cuadernos de la Cátedra "la Caixa" de Responsabilidad Social de la Empresa y Gobierno Corporativo. № 1 , 2008.

BESSERA, T. y MILLER, N. Is the good corporation dead? The community social responsibility of small business operators. Journal of Socio-Economics, Vol. 30 No. 3, pp. 221-42, 2001.

CARROLL, A. B. y SHABANA, K.M. The business case for corporate social responsibility: A review of concepts, research and practice. International Journal of Management Reviews, vol. 12, no. 1, pp. 85-105, 2010.

CASTKA, P., BALZAROVA, M. A., BAMBER C. J., y SHARP, J. M. How can SMEs effectively implement the CSR agenda? a UK case study perspective. Corporate Social Responsibility $\&$ Environmental Management. Vol. 4, No 11(3), pp. 140-149, 2004. 
CILIBERTI, F., PONTRANDOLFO, P. y SCOZZI, B. "Investigating corporate social responsibility in supply chains: a SMEs perspective". Journal of Cleaner Production, vol. 16, pp. 1579-1588, 2008.

COM (2001) 681 final: Libro blanco de la Comisión Europea. Un nuevo impulso para la juventud europea. Bruselas. En línea: http://www.cucid.ulpgc.es/documentos/1-

documentos/3/libroblanco.pdf

CRANE, A., MATTEN, D., y SPENCE, L. J. Corporate social responsibility in a global context. Abingdon: Routledge, 2013.

DHILLON, G. S. Understanding social responsibility issues in the information age. Hershey, PA: Idea Group Publishing, 2002.

DU, S., BHATTACHARYA, C. B. y SEN, S. Maximizing business returns to Corporate Social Responsibility (CSR): The role of CSR communication. International Journal of Management Reviews, vol. 12, no. 1, pp. 8-19, 2010.

ELKINGTON, J. Cannibals with forks. The triple bottom line of 21st century. Capstone, 1997.

FRYNAS, J. G., y YAMAHAKI, C. Corporate social responsibility: review and roadmap of theoretical perspectives. Business Ethics: A European Review, 25(3), pp. 258-285, 2016.

FULLER, T. y TIAN, Y. Social and Symbolic Capital and Responsible Entrepreneurship: an empirical investigation of SME narratives. Journal of Business Ethics. Vol. 67, pp. 287-304, 2006.

GALLARDO-VÁZQUEZ, D., SÁNCHEZ-HERNÁNDEZ, M. I., y CORCHUELO-MARTÍNEZAZÚA, M. B. Validación de un instrumento de medida para la relación entre la orientación a la Responsabilidad Social Corporativa y otras variables estratégicas de la empresa. Revista de Contabilidad, 16(1), pp. 11-23, 2013.

GARCÍA-BORBOLLA, A., HERRERA, J., LARRÁN, M., SÁNCHEZ, G. y SUÁREZ, A.: Análisis empírico de la influencia de la propiedad familiar sobre la orientación estratégica de las pequeñas y medianas empresa. Investigaciones Europeas de Dirección y Economía de la Empresa, 15(1), pp. 45-59, 2009.

JAMALI, D., ZANHOUR, M. y KESHISHIAN, T. Peculiar strengths and relational attributes of SMEs in the context of CSR. Journal of Business Ethics, vol. 87, no. 3, pp. 355-377, 2009.

JAMALI, D., SAFIEDDINE, A. M., y RABBATH, M. Corporate governance and corporate social responsibility synergies and interrelationships. Corporate Governance: An International Review, 16(5), pp. 443-459, 2008.

JENKINS, H. Small business champions for Corporate Social Responsibility. Journal of Business Ethics, vol. 67, no. 3, pp. 241-256, 2006.

Jenkins, H.: A critique of conventional CSR theory: An SME perspective. Journal of General Management, vol. 29, $\mathrm{n}^{\circ}$. 4, pp: 37-57, 2004.

JONKER, J. y de WITTE, M. (eds). Management models for Corporate Social Responsibility. Berlin: Springer, 2006.

KECHICHE, A. y SOPARNOT, R. "CSR with SMEs: Literature review”. International Business Research, vol. 5, no. 7, pp. 97-104, 2012. 
KELL, G.: The Global Compact: Origins, Operations, Progress, Challenge. Journal of Corporate Citizenship, Vol 11, pp. 35-49, 2003.

KUSYK, S. M. y LOZANO, J. M. Corporate responsibility in small and medium-sized enterprises SME social performance: a four-cell typology of key drivers and barriers on social issues and their implications for stakeholder theory. Corporate governance. Vol. 7, nº 4, pp. 502-515, 2007.

LANGE, E. A., y FENWICK, T. J. Moral commitments to community: mapping social responsibility and its ambiguities among small business owners. Social Responsibility Journal, 4(1/2), pp.41-55, 2008.

LEE, M. H., MAK, A. K. y PANG, A. Bridging the gap: An exploratory study of Corporate Social Responsibility among SMEs in Singapore. Journal of Public Relations Research, vol. 24, no. 4, pp. 299-317, 2012.

LEE, K. H., HEROLD, D. M., y YU, A. L. (2015). Small and medium enterprises and corporate social responsibility practice: A Swedish perspective. Corporate Social Responsibility and Environmental Management. En línea: https://www.researchgate.net/profile/David

Herold4/publication/272201141_Small_and_Medium_Enterprises_and_Corporate_Social_Responsibil ity_Practice_A_Swedish_Perspective/links/57059ff608aef745f7176e8f.pdf

LEPOUTRE, J. y HEENE, A. Investigating the impact of firm size on small business social responsibility: a critical review. Journal of Business Ethics, vol. 67, no. 3, pp. 257-273, 2006.

LINDGREEN, A, SWAEN, V. y MAON, F. Introduction: Corporate Social Responsibility implementation. Journal of Business Ethics, vol. 85, pp. 251-256, 2009.

MADUEÑO, J. H., JORGE, M. L., SANCHO, M. P. L. y MARTÍNEZ-MARTÍNEZ, D. Responsabilidad social en las pymes: análisis exploratorio de factores explicativos. Revista de Contabilidad, 19(1), pp. 31-44, 2016.

MAON, F., LINDGREEN, A. y SWAEN, V. Designing and implementing Corporate Social Responsibility: An integrative framework grounded in theory and practice. Journal of Business Ethics, vol. 87, no. 1, pp. 71-89, 2009.

MARTÍNEZ, J. y AGÜERO, A. The why, when, and how of corporate social responsibility. Journal of strategic management education, 4(16493877), pp. 117-133, 2008.

MASON, C. y SIMMONS, J. Embedding corporate social responsibility in corporate governance: A stakeholder systems approach. Journal of Business Ethics, 119(1), pp. 77-86, 2014.

McELHANEY, K.: Just Good Business; The strategic guide to aligning corporate responsibility and brand. San Francisco: Berrett-Koehler Publishers, Inc, 2008.

MURILLO, D y LOZANO, J. M. SMEs and CSR: An Approach to CSR in their own words. Journal of Business Ethics, vol. 67, pp. 277-240, 2006.

PANG, A., MAK, A. y LEE, J. Significance of sector-specific Corporate Social Responsibility Initiatives-Status and role in different sectors, Bartlett, J.L. y May S. (eds), The handbook of communication and Corporate Social Responsibility, Wiley-Blackwell, Oxford, pp. 295-314, 2011.

PERRINI, F. SMEs and CSR theory: Evidence and implications from an Italian Perspective. Journal of Business Ethics, vol. 67, no. 3, pp. 305-316, 2006. 
PORTER, M. E. y KRAMER, M. R. The link between competitive advantage and Corporate Social Responsibility. Harvard Business Review, vol. 84, no. 12, pp. 78-92, 2006.

RIVES, L. M., y BAÑÓN, A. R. La responsabilidad social corporativa como determinante del éxito competitivo: un análisis empírico. Revista europea de dirección y economía de la empresa, 17(3), pp. 27-42, 2008.

RUSSO, A. y PERRINI, F. Investigating Stakeholder Theory and social capital: CSR in large firms and SMEs. Journal of Business Ethics, vol. 91, no. 2, pp. 207-221, 2010.

RUSSO, A. y TENCATI, A. Formal vs. informal CSR strategies: Evidence from Italian micro, small, medium-sized, and large firms. Journal of Business Ethics, vol. 85, no. 2, pp. 339-353, 2009.

SAVITZ, A. W. y WEBER, K.: The triple bottom line. San Francisco, Jossey-Boss, p. 320, 2006.

SCHAPER, M. y SAVERY, L. Entrepreneurship and philanthropy: the case of small Australian firms. Journal of Development Entrepreneurship, Vol. 9 No. 3, pp. 239-50, 2004.

SPENCE, L. J. y RUTHERFOORD, R. Social responsibility, profit maximization and the small firm owner-manager. Journal of Small Business and Enterprise Development, vol. 8, no. 2, pp. 126-139, 2000.

SPENCE, L. J. y SCHMIDPETER, R. SMEs, social capital and the common good. Journal of Business Ethics, vol. 45, no. 1-2, pp. 93-108, 2003.

SPENCE, L. J. Does size matter? The state of the art in small business ethics. Business Ethics: A European Review, vol. 8, no. 3, pp. 163-174, 1999.

SPENCE, L. J. CSR and small business in a European policy context: The five "C"s of CSR and small business research agenda 2007. Business y Society Review, vol. 112, no. 4, pp. 533-552, 2007.

SWEENEY, L. Corporate Social Responsibility in Ireland: Barriers and opportunities experienced by SMEs when undertaking CSR. Journal of Corporate Governance, vol. 7, no. 4, pp. 516-523, 2007.

SWEENEY, L.: A study of current practice of corporate social responsibility (CSR) and an examination of the relationship between CSR and financial performance using structural equation modelling (SEM). Dublin: Institute of Technology, 2009.

TANTALO, C., CAROLI, M. G. y VANEVENHOVEN, J. Corporate social responsibility and SME's competitiveness. International Journal of Technology Management, 58(1/2), pp.129-151, 2012.

VIRVILAITE, R. y DAUBARAITE, U. Corporate social responsibility in forming corporate image. Engineering Economics, 22(5), pp. 534-543, 2015.

VIVES, A. Social and environmental responsibility in Small and Medium Enterprises in Latin America. Journal of Corporate Citizenship, vol. Spring, no. 21, pp. 39-50, 2006.

WEBB, E.: An examination of socially responsible firms' board structure. Journal of Management and Governance, 8, pp.255-277, 2004. 
Profesora Doctora del Departamento de Administración y Direción de Empresas. Profesor Doctor del Departamento de Ciencias Jurídicas y de la Empresa 